\title{
HIPEREMESIS GRAVÍDICA Y PESO DEL RECIÉN NACIDO. INSTITUTO NACIONAL MATERNO PERINATAL. AÑO 2008
}

\author{
Edwin Elard Cabrera Arroyo ${ }^{1}$, Karen Evelyn Cabrera Luna ${ }^{2}$
}

\begin{abstract}
RESUMEN
Objetivos. Determinar la relación de la hiperemesis gravídica sobre el peso del recién nacido en el Instituto Nacional Materno Perinatal durante el 2008. Materiales y métodos. Se realizó un estudio correlacional retrospectivo, en 120 gestantes con hiperémesis gravídica comprendidos en el periodo enero a diciembre del 2008 atendidos en éste instituto. Se utilizó la técnica de observación documental en historias clínicas, indagando sobre las características generales, maternas, y del recién nacido. El análisis de los datos se realiza a través de estadísticas descriptivas e inferenciales con prueba de hipótesis significativas a un nivel de significación estadístico $p<0,05$. Resultados. La incidencia de hiperémesis gravídica es de 0,69; la edad promedio de las gestantes evaluadas es de $27,3 \pm 6,2$; talla media de $1,54 \pm 0,06$; peso pregestacional promedio $58,4 \pm 7,9$; índice de masa corporal promedio $24,6 \pm 3,2$; nivel de hemoglobina medio $12,7 \pm 1,2$. De los resultados evaluados se demuestra que los promedios de pesos del RN en el grado de severo y moderado de la hiperémesis gravídica son similares, existiendo evidencias estadísticas con un nivel de confianza del 95\% para afirmar que no existe relación entre las variables peso del recién nacido y el grado de severidad de la presencia de hiperémesis gravídica p=0,83. Conclusión. Los efectos de la hiperémesis gravídica no tienen relación sobre el peso del recién nacido.
\end{abstract}

Palabras clave: Hiperemesis gravídica; Peso del recién nacido (fuente: DeCS BIREME).

\section{HYPEREMESIS GRAVIDARUM AND BIRTH WEIGHT. MATERNAL PERINATAL NATIONAL INSTITUTE. YEAR 2008}

\begin{abstract}
Objectives. To determine the relation between hyperemesis gravidarum and the newborn weight at Maternal Perinatal National Institute during 2008. Materials and methods. A correlational retrospective study was carried out in 120 pregnant women with hyperemesis gravidarum who were seen from January to december in 2008 at this institute. A documentary observation method was utilized in the clinical records, and some investigation about the general characteristics involving mothers and newborns was also made. The datum were analyzed through significant descriptive and inferential statistics with hypothesis testing at a level of statistical significance $p<0,05$. Results. The hyperemesis gravidarum incidence is 0,69 . The average age of the pregnant women that were evaluated is $27,3 \pm 6,2$; an average height of $1,54 \pm 0,06$; an average pregestational weight of $58,4 \pm 7,9$; an average body mass index of $24,6 \pm 3,2$; and an average hemoglobin level of $12,7 \pm 1,2$. From the evaluation of the results, it is clear that the average weights of the newborns in the severe and moderate hyperemesis gravidarum are similar. There is statistical evidence at a $95 \%$ confidence level to affirm that there is not any relation between these variables: the newborn weight and the severity degree of hyperemesis gravidarum $p=0,83$. Conclusion. The effects of hyperemesis gravidarum do not have any relation with the newborn weight.
\end{abstract}

Key words: Hyperemesis gravidarum; Newborn weight (source: MeSH NLM).

\section{INTRODUCCIÓN}

La gran mayoría de gestantes experimentan algún tipo de náuseas o vómitos durante el transcurso de sus embarazos. A los mismos, comúnmente se los conoce como emesis gravídica, y estos signos propios del embarazo, por lo general, no causan serias complicaciones en su salud ni en la del feto.

No obstante, si estuviera experimentando persistentes náuseas y vómitos podría estar padeciendo una forma más severa, conocida como hiperémesis gravídica; complicación que se define como la presencia de náuseas y vómitos severos, que producen deshidratación, pérdida de peso mayor del $5 \%$, cetonuria y transtornos metabólicos y electrolíticos (Gross, Librach \& Cecutti ${ }^{8}$ 1990; Koubaa, Hällström, Lindholm ${ }^{13}$, et al, 2005; Tan, Jacob, Quek \& $\mathrm{Omar}^{23}$, 2007). Se considera a la hiperémesis gravídica severa aquella que presenta signos de deshidratación severa, ansiedad, ictericia, cetoacidosis, pérdida de peso mayor al $10 \%$, oliguria y en ocasiones shock. (Eliakim ${ }^{6}$ 2000; Guerra, Vilela ${ }^{9}$ 2006) o al menos uno de los siguientes criterios: cetonuria, incremento de urea sérica y hematocrito y/o electrolitos anormales (HallaK M ${ }^{10}$ 1996).

Los síntomas se inician a las cuatro semanas y se extienden hasta las 12 y 15 semanas de gestación. Alrededor del $50 \%$ de las pacientes con náuseas y vómito

\footnotetext{
Médico GinecoObstetra, Asistente del Departamento de Obstetricia del Instituto Nacional Materno Perinatal. Magíster en Salud Pública. Profesor Asociado de la Facultad de Medicina Humana "Hipólito Unanue" de la Universidad Nacional Federico Villarreal. Lima - Perú.

2 Médico Cirujano. Egresado de la Facultad de Medicina Humana de la Universidad Ricardo Palma. Lima - Perú
} 
en el embarazo tienen problemas laborales, el $25 \%$ no trabajan durante la enfermedad; un $50 \%$ alteran sus relaciones sociales y $55 \%$ sentirán depresión.

Poco se sabe también, sobre el curso del embarazo y la salud de los hijos de madres con trastornos asociados a la ingesta de alimentos como la hiperémesis gravídica. Los hallazgos de los estudios publicados a la fecha sugieren que esta entidad afectaría negativamente los resultados fetales, habiéndose asociado con parto pretermino, restricción del crecimiento intrauterino, bajo peso al nacer y Apgar bajo al nacer (1,2 Katz, Vollenhoven ${ }^{12}, 2000$; Morrill \& Nickols-Richardson ${ }^{18}$, 2001). Sin embargo, los reportes de casos también muestran recién nacidos con peso normal y ausencia de complicaciones médicas (Dodds, Fell, Joseph \& Allen 5 , 2006; Gross, Librach \& Cecutti $\left.^{8}, 1990\right)$. Tan et $a^{23}$ en un estudio retrospectivo realizado entre enero 2004 - 2005, en 165 mujeres hospitalizadas por hiperémesis gravídica, y publicado en el J Obstet Gynaecol Res 2007, para determinar los resultados de gestantes con éste trastorno y los efectos de los indicadores bioquímicos, hematológicos y clínicos sobre la severidad de los resultados óbito fetal, Apgar al nacer, vía del parto, bajo peso al nacer, parto pretérmino, inducción del parto, trastornos hipertensivos y diabetes gestacional. Las gestantes con hiperémesis tuvieron resultados similares comparados con los controles. Concluyeron que la hiperémesis gravídica per se no se asoció con resultados obstétricos adversos. La hipokalemia, creatinina elevada y el incremento de la gamma glutamiltransferasa en gestantes con hiperémesis sí se asoció con resultados obstétricos desfavorables.

Sin embargo, Koubaa et al ${ }^{13}$ en un estudio realizado el 2005 (Pregnancy and neonatal outcomes in women with Ealing disorders), para evaluar los resultados de la gestación y neonatales en 49 mujeres con historia de trastornos de la alimentación pasados o actuales comparados con un grupo control de 68 gestantes. El $22 \%$ de las pacientes presentó una recaída en los trastornos de la alimentación durante el embarazo. Las mujeres con historia de trastornos de la alimentación presentaron mayor riesgo de hiperémesis y tuvieron neonatos con peso al nacer y circunferencia cefálica significativamente menores comparadas con los controles.

También presentaron mayor riesgo de tener neonatos pequeños para la edad gestacional y microcefalia. Concluyeron que las gestantes con trastornos de la alimentación parece que tienen mayor riesgo de neonatos pequeños para la edad gestacional, bajo peso al nacer, disminución de la circunferencia cefálica y microcefalia.

Paauw et al ${ }^{19}$ en una investigación publicada en el JPEN $J$ parenter Enteral Nutr 2005 (Hyperemesis gravidarum and fetal outcome), realizaron un estudio prospectivo de tipo cohorte con 45 pacientes diagnosticadas con hiperémesis gravídica comparadas con 306 gestantes sin hiperémesis con embarazos únicos, con el objetivo de evaluar la relación entre hiperémesis gravídica materna, peso al nacer y resultados neonatales. Concluyeron que los neonatos de madres que cursaron con hiperemesis gravídica experimentaron disminución de la edad gestacional e incremento de la estancia hospitalaria y recomendaron que deba estudiarse la eficacia del tratamiento precoz y agresivo de la hiperémesis gravídica, incluyendo soporte nutricional para minimizar estos resultados.

El objetivo del presente estudio fue determinar la relación de la hiperemesis gravídica sobre el peso del recién nacido atendidas durante el período entre el 01 de enero y el 31 de diciembre del 2008 en el Instituto Nacional Materno Perinatal (INMP).

\section{MATERIALES Y MÉTODOS}

Se realizó un estudio descriptivo, correlacional retrospectivo, transversal, donde se seleccionaron 120 casos de hiperémesis gravídica atendidos en el periodo enero a diciembre del 2008 en el Instituto Nacional Materno Perinatal donde se registraron en total 49950 gestantes y 17392 partos. Se realizó la técnica de observación documental en historias clínicas, a los cuales se indagó sobre características generales, maternas, de la hiperémesis y del recién nacido. Posteriormente se confeccionó una base de datos en Microsoft Excel y se analizó a través de estadísticas descriptivas e inferenciales con prueba de hipótesis significativas a un nivel de significación estadístico $p<0,05$.

Criterios de inclusión: Síndrome de hiperémesis gravídica atendida en el Instituto Nacional Materno Perinatal durante el periodo 2008 y cuyos registros de historia clínica fueron completos.

Criterios de Exclusión: Preeclampsia, diabetes mellitus, infecciones urinarias, cardiopatías, periodo internatal corto, anemia crónica, embarazo múltiple, óbito fetal, parto pretérmino, embarazo molar y acretismo placentario

\section{RESULTADOS}

El presente estudio de investigación se realizó evaluando a 120 gestantes que presentaron el síndrome de hiperémesis gravídica (HG) en el Instituto Nacional Materno Perinatal durante el año 2008, según los criterios de inclusión y exclusión establecidos en el proyecto de investigación, es decir, la incidencia de hiperémesis gravídica es de 0,69.

El perfil demográfico de las gestantes es: en su mayoría de edad fértil $(76,7 \%)$, con nivel de estudios secundario $(60 \%)$ y estado civil conviviente $62,5 \%$.

La procedencia de donde residen en su mayoría (45,8\%) son de San Juan de Lurigancho 17,5\%, San Juan de Miraflores $13,3 \%$, Rímac 7,5\% y cercado de Lima 7,5\%. 
Tabla 1. Relación entre hiperémesis gravídica y peso al nacer INMP-2008

\begin{tabular}{|c|c|c|c|c|c|c|c|}
\hline & & \multicolumn{4}{|c|}{ Hiperemesis gravídica } & \multirow{2}{*}{\multicolumn{2}{|c|}{ Total }} \\
\hline & & \multicolumn{2}{|c|}{ Moderada } & \multicolumn{2}{|c|}{ Severa } & & \\
\hline & & $\mathbf{N}$ & $\%$ & $\mathbf{N}$ & $\%$ & $\mathbf{N}$ & $\%$ \\
\hline \multirow{5}{*}{$\begin{array}{l}\text { Peso } \\
\text { del RN }\end{array}$} & $<2500$ & 2 & $3,0 \%$ & 2 & $3,7 \%$ & 4 & $3,3 \%$ \\
\hline & [ $2500-3000$ ] & 12 & $18,2 \%$ & 11 & $20,4 \%$ & 23 & $19,2 \%$ \\
\hline & $<3000-4000]$ & 47 & $71,2 \%$ & 39 & $72,2 \%$ & 86 & $71,7 \%$ \\
\hline & $>4000$ & 5 & $7,6 \%$ & 2 & $3,7 \%$ & 7 & $5,8 \%$ \\
\hline & Total & 66 & $100,0 \%$ & 54 & $100,0 \%$ & 120 & $100,0 \%$ \\
\hline
\end{tabular}

$X^{2}=0,882(p=0,83)$

La edad promedio de las gestantes evaluadas es de 27,3 \pm 6,2 , talla media de $1,54 \pm 0,06$, peso pregestacional promedio $58,4 \pm 7,9$, índice de masa corporal promedio $24,6 \pm 3,2$, nivel de hemoglobina medio $12,7 \pm 1,2$. Como dato adicional es la estancia hospitalaria promedio fue de $5,1 \pm 2,3$.

Otras características maternas importantes de describir es: en su mayoría son Multíparas $(60,83 \%)$, Nuliparidad (39,2\%), $78,3 \%$ de talla materna entre $1,45-1,60 \mathrm{~m}, 55 \%$ con peso normal y $37,5 \%$ con sobrepeso, $51,7 \%$ fueron cesareadas, $4,2 \%$ se indujo el parto, sólo el $9,2 \%$ se consideran anémicas, días de estancia hospitalaria $60 \%$ entre 4 a 7 días, $24,2 \%$ menores a 4 días y con estancia prolongada $15,9 \%$. De los que se realizaron examen de orina el $15 \%$ resultaron ser patológicos.

De los resultados evaluados en la tabla 1 se demuestra que existe evidencias estadísticas con un nivel de confianza del $95 \%$ para afirmar que no existe relación entre las variables peso del RN y el grado de severidad de la presencia de hiperémesis gravídica $p=0,83$. El resultado analítico podemos observar en la figura 2, así tenemos que la frecuencia relativa entre el grado severo $3,7 \%$ y moderado $3 \%$ son muy próximos para recién nacidos menores de $2500 \mathrm{~g}$; el porcentaje entre el grado severo $20,4 \%$ y moderado $18,2 \%$ son muy próximos para recién nacidos entre de 2500 a $3000 \mathrm{~g}$; el porcentaje entre el grado severo $72,2 \%$ y moderado $71,2 \%$ son

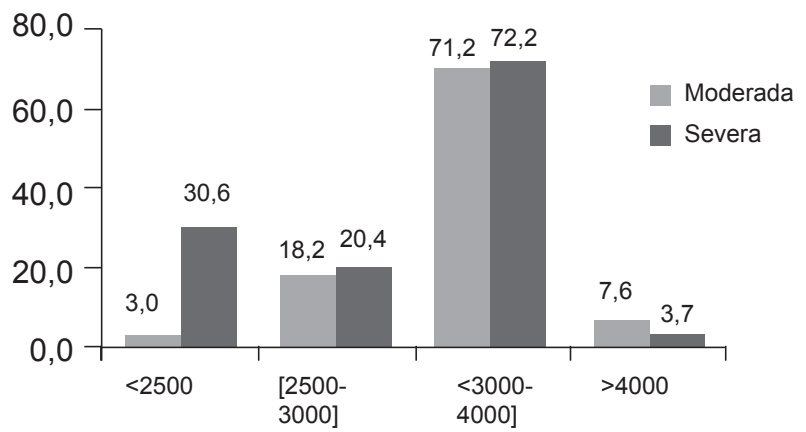

Figura 2. Comparativo entre grados de hiperemesis gravídica y categorías de peso al nacer INMP-2008
Tabla 3. Correlación entre hiperémesis gravídica y peso al nacer INMP-2008

\begin{tabular}{|c|c|c|c|c|}
\hline & & & $\begin{array}{l}\text { Hiperemesis } \\
\text { gravídica }\end{array}$ & $\begin{array}{c}\text { Peso al } \\
\text { nacer }\end{array}$ \\
\hline \multirow{6}{*}{$\begin{array}{l}\text { Rho de } \\
\text { Spearman }\end{array}$} & \multirow{3}{*}{$\begin{array}{l}\text { Hiperémesis } \\
\text { gravídica }\end{array}$} & $\begin{array}{l}\text { Coeficiente } \\
\text { de correlación }\end{array}$ & 1,000 &,- 174 \\
\hline & & Sig. (bilateral) & & ,058 \\
\hline & & $\mathrm{N}$ & 120 & 120 \\
\hline & \multirow{3}{*}{$\begin{array}{l}\text { Peso al } \\
\text { nacer }\end{array}$} & $\begin{array}{l}\text { Coeficiente } \\
\text { de correlación }\end{array}$ &,- 174 & 1,000 \\
\hline & & Sig. (bilateral) & ,058 & \\
\hline & & $\mathrm{N}$ & 120 & 120 \\
\hline
\end{tabular}

muy próximos para recién nacidos entre de 3000 a 4000 g. Para los mayores de $4000 \mathrm{~g}$ al parecer existe cierta diferencia pero sólo es descriptiva $3,7 \%$ y $7,6 \%$ entre el grado severo y moderado respectivamente.

Con la finalidad de confirmar si realmente existe alguna posibilidad de relación entre estas dos variables, podemos utilizar el coeficiente de correlación estadístico ${ }^{1}$ entre estas dos variables. Como la variable hiperémesis gravídica es una variable ordinal y el peso del RN una variable numérica, lo adecuado es utilizar el coeficiente de correlación de Spearman.

Los promedios de pesos del RN en el grado de severo y moderado de la hiperémesis gravídica son similares (EI rango intercuartilico entre ambos diagramas de caja son muy próximos, entre 3000 y $3600 \mathrm{~g}$ aproximadamente)

\section{DISCUSIÓN}

La hiperémesis gravídica es una condición poco frecuente asociada al embarazo, sin embargo, representa un alto riesgo materno-fetal. Las tasas de incidencia son variables, en nuestro estudio encontramos una incidencia de 0,69 \%. En una revisión hecha por Eliakim ${ }^{6}$ (2000) se reporta una notable variación entre $0,04 \%$ hasta 3,03\% de todos los nacidos vivos en Estados Unidos. Este

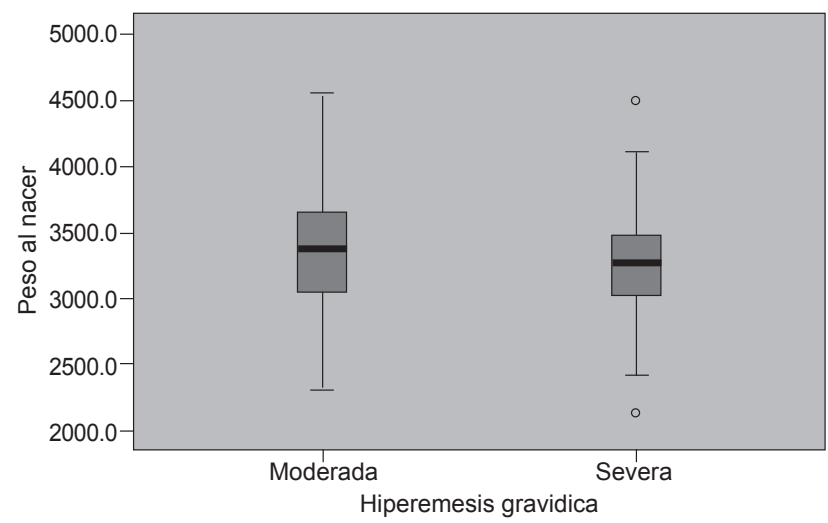

Figura 4. Comparativo entre grados de hiperemesis gravídica y peso al nacer INMP-2008 
mismo autor, menciona una cifra de $1,48 \%$ para Gran Bretaña, 1\% para Europa Continental y 0,19\% para AsiaÁfrica. Fitzgerald citado por Iffy ${ }^{25}$ (1996), en un estudio comunitario en Aberdean basado en la población total, reveló una incidencia de $1,02 \%$, mientras que en Burdeos, la incidencia fluctuó entre $0,62 \%$ y $0,65 \%$ en un estudio de 6 años.

Toda esta variabilidad se explica, porque no siempre los criterios diagnósticos son respetados escrupulosamente, así como la falta de una definición clara de hiperemesis y quizás la recolección de datos de poblaciones altamente seleccionadas.

En nuestro estudio, la edad de las pacientes varió entre 20 a 35 años con una Desviación estándar (SD) de 27,3 \pm 6,2 años, similar a lo observado por Depue y $\mathrm{col}^{4}$ (1987). En relación con la edad Iffi25 (1996), presenta una significativa disminución de la incidencia de HG al aumentar la edad materna.

En la presente serie hubo un predominio de hiperémesis en las pacientes con estado civil ilegítimo: solteras y unión estable (convivientes), sin embargo, según Mannor ${ }^{17}$ (1990), no hay ninguna asociación significativa entre hiperémesis y el estado civil, ya que más importante que el problema de la legitimidad es la aceptación o el rechazo al embarazo. El embarazo extramatrimonial no tiene por qué ser sinónimo de rechazo; existen muchas embarazadas solteras que sobrellevan muy bien su gestación, no presentando manifestaciones psico-somáticas.

La influencia de la paridad ha arrojado resultados contradictorios, ya que para algunos autores es factor influyente, pero para la mayoría, carece de absoluta importancia (Koubaa ${ }^{13}$ (2005), es así como nuestra investigación se presenta con mayor frecuencia entre el segundo y quinto embarazo, con un promedio de 2 . Otros autores como, Iffy ${ }^{25}$ (1996) han informado un ligero aumento en primíparas.

La mayoría de las hospitalizaciones ocurre entre la semana 8 y 12 de la gestación, durante 2 a 15 días con una desviación estándar de 5,1 \pm 2,3 coincidiendo con Mannor $^{17}(1990)$.

La talla materna de las gestantes estudiadas con hiperémesis gravídica tuvo una media de 1,54 $\pm 0,06$, (variando entre 1,42 y $1,70 \mathrm{~m}$ ) ; peso pregestacional $58,4 \pm 7,9$ (entre 45 y $86 \mathrm{Kg}$ ) y el índice de masa corporal (IMC) con una desviación estándar de 24,6 \pm 3,2 (entre 18,25 y -33,59) demostrando que se encontraban en peso y talla normales, lo que no influyó en un peso bajo del recién nacido. En el feto, la malnutrición puede dificultar su formación y desarrollo en caso de graves carencias en su etapa de hiperplasia y en su etapa de hipertrofia y desarrollo, según Pacheco ${ }^{20}$ (1990).

A pesar de que la hiperémesis gravídica representa un alto riesgo materno fetal, la mayoría de nuestras pacientes culminaron el embarazo a término, siendo la vía del parto por cesárea la más frecuente $(51,7 \%)$.

La hemoglobina se encontró en valores entre 9,9 - 15,6 g con una desviación estándar de 12,7 \pm 1,2 debido a la hemoconcentración por la deshidratación severa materna.

La relación de la hiperémesis con el feto, se ha investigado según el peso al nacimiento, así encontramos estudios realizados por Koubaa et al ${ }^{13}$ (2005), Paauw et $\mathrm{al}^{19}$ (2005) Depue et al $(1987)$, Dodds, Fell, Joseph, et a/ ${ }^{5}$ (2006) quienes concluyeron que las madres que tuvieron el síndrome de hiperémesis gravídica tenían mayor probabilidad de tener recién nacidos de bajo peso al nacer.

Por el contrario Tan et $a^{23}$ entre enero 2004 - diciembre 2005, Jarnfelt-Samsoie et al ${ }^{11}$ (1983) encontraron que la hiperémesis gravídica per se no se asoció con resultados obstétricos adversos, no encontrando diferencias significativas entre los pesos de los recién nacidos, con los hijos de madres que no tuvieron la complicación de hiperémesis gravídica durante el embarazo. Es así como, en nuestra investigación, los pesos de los recién nacidos encontrados, en los casos de hiperémesis moderada y severa, está en el rango de normalidad, $(71,7 \%$ entre 3000 a $4000 \mathrm{~g}$ ).

Se puede concluir que los efectos de la hiperemesis gravídica no tienen relación sobre el peso del recién nacido.

Es recomendable realizar la investigación pero identificando un grupo control adecuado, y comparativo con otras instituciones de salud

\section{REFERENCIAS BIBLIOGRÁFICAS}

1. Bashiri A, Neumann, Katz M. Hiperemesis gravidarum: epidemiologic features, complications and outcome. Eur J Obstet Gynecol Reprod Biol, 1995; 63(2):135-8.

2. Chin RK, Lao TT. Low birth weight and hyperemesis gravidarum. Eur J Obstet Gynecol Reprod Biol 1988;28(3): 179-83.

3. Chin . Hyperemesis Gravidarum and fetal growth retardation. Am J. Obstet Gynecol 1989;160(4): 6-9.

4. Depue R, Bernstein L, Ross R Judd H, Henderson B. Hyperemesis gravidarum in relation to estradiol levels, pregnancy outcome and other maternal factors: A seroepidemiologic study. Am J Obstet Gynecol 1987;156: 1137-1141.

5. Dodds L, Fell DB, Joseph KS, Allen VM. Outcomes of pregnancies complicated by hyperemesis gravidarum. Obstet Gynecol 2006;107 (2 Pt ): 285-92.

6. Eliakim R, Abulafia $O$, Sherer D. Hyperemesis gravidarum: $A$ current review. Am J Perinatol, 2000;17: 207-218.

7. Folk J, Leslie-Brown H, Silverman R, Aubry R. Hyperemesis gravidarum: outcomes and complications with and without total parenteral nutrition. J Reprod Med, 2004;49: 497-502.

8. Gross S, Librach C, Cecutti A. Maternal weight low associated with hiperemesis gravidarum: a predictor of fetal outcome. Am J Obstet Gynecolgy 1990;160: 906-909. 
9. Guerra Luis, Vilela O. Guía Practica Clínica SOAR- HNGAI Essalud 2006: 77.

10. Hallak M, TsalamandrisK, Dombrowski MP, Isada NB. Hyperemesis gravidarum.effects on fetal outcome. J Reprod Med 1996;41(11):871-4

11. Jarnfelt-Samsoie A, Samsoie G. Nausea and vomiting in pregnancy: A contribution to its epidemiology. Gynecol Obstet Invest 1983;16:221-229.

12. Katz MG, Vollenhoven B. The reproductive endocrine consequences of anorexia nervosa. BJOG 2000;107: 707-13.

13. Koubaa S, Kouba S, Hällström T, Lindholm C. Pregnancy and neonatal outcomes in women with eating disorders. Obstet Gynecol 2005;105 (2): 255-60.

14. Kramer MS. Determinants of low birth weight: methodological assessment and meta-analysis. Bull World Heath Organ 1997;65(5):663-737.

15. Kruse $\mathrm{H}$, Adomssent $\mathrm{S}$, Herre $\mathrm{H}$. Body measurements and morphological signs of maturity in newborn infants after hyperemesis gravidarun. Zentralbl Gynacol, 1995;97(18):105-9.

16. López I, Lugones IM, Valdespino L. Algunos factores maternos relacionados con el bajo peso al nacer. Rev Cubana Obstet Ginecol 2004;30(1).

17. Mannor SM (1990). Hiperemesis gravídica. En: Iffy L; Kaminetzky HA. Obstetricia y perinatología. Buenos Aires, Argentina. Ed. Panamericana.
18. Morrill ES, Nickols-Richardson HM. Bulimia nervosa during pregnancy: a review. J Am Diet Assoc 2001;101: 448-54.

19. Paauw JD, Bierling S, Cook CR, Davis AT. Hyperemesis gravidarum and fetal outcome. JPEN J Parenter Enteral Nutr 2005;29 (2):93-6.

20. Pacheco J. Importancia del espaciamiento de los nacimientos. Salud Materna y Perinatal. Red Peruana de Perinatología, Edit. San Miguel, Lima, 1990.

21. Philip B. Hyperemesis gravidarum: Literature review. Wis Med J 2003;102: 46-51.

22. Sugito Y, Sekizawa A, Farina A, Yukimoto Y, et al. Relationship between severity of hyperemesis gravidarum and fetal DNA concentration in maternal plasma. Clin Chem 2003; 49: $1667-$ 1669.

23. Tan PC, Jacob R, Quek KF, Omar SZ. Pregnancy outcome in hyperemesis gravidarum and the effect of laboratory clinical indicators of hyperemesis severity. J Obstet Gynaecol Res 2007;33 (4): 457-64.

24. Tsang IS, Katz VL, Wells SD. Maternal and fetal Outcomes in Hiperemesis gravidarum. Int J Gynaecol Obstet 1996;55 (3) 231-5.

25. Iffy J, Taylor. Succesful management of hiperemesis gravidarum using steroid therapy. OJM 1996; 89:103-107 